\title{
El universo y el gran diseño de Dios, un acercamiento desde la ciencia y la teología
}

\author{
Pedro J. Ramírez Acosta*
}

Recibido: Julio 2011 • Aceptado: Setiembre 2012

RESUMEN

El presente ensayo busca indagar y reflexionar sobre el gran diseño cosmológico, preocupación tanto de la filosofía y de la ciencia como de la teología. En una primera parte, el autor analiza el desarrollo, la búsqueda y respuestas de la ciencia, en especial, de la física teórica. Y en una segunda parte, rastrea el camino de la revelación y de la fe en busca del gran diseño cosmo-teológico integrador, que es la historia de la salvación. Reconoce que el orden del infinito océano cósmico está orientado al hombre y que toda la creación solo tiene sentido en Cristo, centro gravitacional y pleroma de todo lo que existe.

Palabras Clave: Diseño cosmológico, Ciencia, Teología, Física teórica, Big Bang, Revelación, Fe y razón, Centro gravitacional, Cosmos, Origen del universo, Teoría geocéntrica, Leyes positivas, Punto Omega, Pleroma.

\begin{abstract}
The present essay seeks to ask and reflect on the big cosmological design, concerning as the philosophy and the science as the theology. In the first part, the author analyzes the development, the search, and the answers of the science, especially of the theoretical physics. In the second part, he goes beyond the way of the revelation and the faith looking for the big cosmo-theological integrator design, which is the history of the salvation. He recognizes that the order of the infinite cosmic ocean is oriented to the man and that all the creation just has a sense in Christ, gravitational center, and pleroma of all what it exists.
\end{abstract}

Key words: Cosmological design, Science. Theoretical physics. Big Bang. Revelation. Faith and Reason. Gravitational center. Cosmos. Universe Origen. Geocentric Theory. Positive laws. Omega Point. Pleroma

* Licenciado en filosofía y en teología por la Universidad de SantoTomás y Gregoriana, Roma, Italia. Máster en Administración de la educación superior por Villanova University, y Doctor en Educación. Catedrático de la UCR y UNED.pra413@gmail.com 
"El movimiento actual de la tierra, el de sus partes y del conjunto, es hacia el centro del universo, de ahí su actual estado en reposo en el mismo".

Aristóteles, Tratado del cielo

"Los cielos cuentan la gloria de Dios, la obra de sus manos anuncia el firmamento..."

Salmo 19, 2

"Te doy las gracias a ti, Dios señor y creador nuestro, porque me dejas ver la belleza de tu creación, y me regocijo con la obra de tus manos. Mira, ya he concluido la obra

a la que me sentí llamado; he cultivado el talento que Tú me diste; he proclamado

la magnificencia de tus obras a los hombres que lean estas demostraciones, en la medida en que pudo abarcarla la limitación de mi espíritu".

Kepler, Armonía del universo

" $M$-theory predicts that a great many universes were creater out of nothing. Their creation does not require the intervention of some supernatural being or god. ....

Although we are puny and insignificant on the scale of the cosmos, this makes us in a sense the lords of creation".

Stephen Hawking, The grand design. pp. 8-9

\section{Introducción}

El tema del diseño del universo es de suma actualidad en medios científicos, religiosos y populares. La cosmología nos presenta hoy un diseño o imagen física del universo diferente a la imagen del universo antiguo y medieval. La cosmología de los griegos daba a conocer un número limitado de esferas, estrellas y planetas; en cambio las hipótesis cosmológicas más recientes señalan que sólo en la Vía Láctea se encuentran un trillón de estrellas y al menos un millón de planetas semejantes al planeta tierra.
Del universo aristotélico finito y centrado en el planeta tierra pasamos a un universo sin centro e ilimitado en extensión. La imagen actual del universo es el resultado de los avances de las ciencias naturales, en especial de la física teórica, y de las nuevas tecnologías.

Por otra parte, la teología nos ofrece otro diseño del universo, en la que espacio, tiempo y personas tuvieron un comienzo y tendrán un final, "el final de los tiempos, en el que todo será nuevo". Desde el Génesis hasta el Apocalipsis las escrituras sagradas reiteran: "Todo el cosmos es obra de las manos de 
Dios". La cosmovisión de las sagradas escrituras es una visión religiosa, no científica. Su máxima preocupación e interés están relacionados con la historia de la salvación de todos los seres humanos. Es, por tanto, una visión lineal de la historia: Dios es alfa y omega, está al inicio, a lo largo y al final de los tiempos.

¿Los grandes avances científicos actuales ofrecen una nueva estructura del universo y conducen a una visión total e integral del cosmos? Para los griegos el cosmos o el universo era la totalidad concebida y ordenada hacia un fin supremo que es el bien. Por tanto, conocer el mundo de las esferas era tan importante como vivir éticamente. El sentido del cosmos incluía el destino de los seres humanos. Tanto las alegrías como el devenir trágico de los seres humanos eran parte de esa antigua racionalidad cósmica. En esta nueva visión científica ¿qué lugar ocupa el hombre, Dios y la teología? ¿Fe y razón, teología y ciencia pueden encontrarse en el camino de la búsqueda de la verdad? ¿Es capaz de integrar y armonizar la totalidad cósmica y humana?

Hawking en su reciente obra The grand design ${ }^{1}$ da a entender que la creación del universo no necesitó

\footnotetext{
1. Es una obra escrita, en 2010, en conjunto con Leonard Mlodinov, doctor en física por la Universidad de Berkeley y escritor de la
}

de un diseñador inteligente que lo sacara de la nada y que más bien los seres humanos, aunque pequeños e insignificantes en la escala del cosmos, son los señores de la creación. ¿Señores de la creación? Señor es una palabra que viene del latín "Dominus" que significa el que tiene poder, el que posee, es soberano, domina y controla. Aclarado el concepto, ¿qué alcances tienen las palabras de este científico? Hawking es un físico teórico de los más prestigiosos del siglo $\mathrm{XX}$, que ha desarrollado planteamientos básicos y medulares de la teoría de la relatividad de Einstein. A diferencia de lo expresado en obras anteriores, como en Historia del tiempo, ¿las nuevas afirmaciones tienen solo un valor mediático?

La sociología de la religión y la teología fundamental actual reconocen un debilitamiento de los patrones religiosos tradicionales, pero igualmente identifican nuevas formas de religiosidad y de espiritualidad en el hombre moderno. Convencido de la necesidad de la ciencia y de la fe, Rahner, refiriéndose a la historia del mundo, en sus Escritos de Teología, identificaba tres categorías fundamentales: Dios

serie Star Trek: The next generation. Es una obra científica con algunos temas clásicos de la filosofía como El misterio del ser, y Qué es la realidad? Y otros que tocan temas fronterizos con la teología, como El milagro aparente y El gran diseño. 
- mundo - historia, estrechamente relacionadas: "La historia de la salvación acontece en la historia del mundo" (T.V., p. 116). Creación, universo, ser humano, gracia vivificante y transformadora son partes esenciales de la teología católica de la historia de la salvación. Dos historias diferentes, la del mundo o profana y la de la salvación, pero implicadas en el marco de un gran diseño, el mejor e insuperable diseño, según Leibniz. Entonces, ¿qué tan cierto es que caminamos hacia una sociedad del conocimiento o de la ciencia sin Dios, donde el universo y sus leyes son el sustituto de Dios y los seres humanos los "Señores de la creación", como señala Howking? Carl Sagan, otro prestigioso físico teórico contemporáneo, se hacía una pregunta más inteligente: ¿Y quién hizo las leyes que gobiernan el universo?

Jamás la sociedad humana alcanzó tanto conocimiento y poder sobre la naturaleza, apoyada en el avance asombroso de las ciencias, en los últimos dos siglos, pero especialmente a partir de la segunda parte del siglo XX con la revolución científico tecnológica. El Renacimiento fue la puerta de entrada a la Edad Moderna. Fue una revolución cultural en casi todos los caminos del conocimiento humano. Desde el arte de Leonardo y Miguel Ángel hasta una nueva visión cósmica ideada por Copérnico. A partir de este momento, la imagen del universo ha cambiado y adquiere nuevos contornos. Sus fronteras se extienden en forma ilimitada en el espacio y en el tiempo. En el campo de la física-matemática, Kepler, Galileo, Newton, De la Mettrie, De Broglie, y más actuales: Heisenberg, Einstein, Hubble, Lemaitre, Hawking, etc., se encargarán de hacer los aportes más significativos. Pero, afirma Rahner: "Ni un individuo, ni una época histórica pueden abrir caminos simultáneamente en todas las direcciones. Por eso, toda conquista implica también una renuncia... Y hay que preguntar a qué se puede renunciar en la conquista, sin que la renuncia sea una maldición mortal" (1967, T. III, p. 429).

En relación con esta renuncia las ciencias de la naturaleza han ido tan lejos en preguntas y respuestas, que han dejado otras fundamentales, largamente meditadas por la teología hebraica cristiana. ¿Por qué existe el universo y no más bien nada? Es la pregunta que se hacía Hawking al final de su obra: Teoría del todo. A diferencia de las afirmaciones actuales, en esta obra su pregunta es fundante y coherente. Lograr una respuesta, afirma, es un triunfo de la razón humana e implicaría entrar a conocer la mente de Dios (2007: 138-139). Producto de esta búsqueda la imagen del universo ha cambiado desde las cosmologías antiguas de Chinos, Sumerios, 
Egipcios, Griegos, y Aztecas hasta nuestros días. Aunque los modelos se han multiplicado y varían unos de otros tanto del macro como del micro cosmos, el universo en su totalidad sigue asombrando y dejando al hombre de ciencia sin una respuesta definitiva. La luz de la ciencia aún no desvela el misterio de la mente de Dios, es decir el diseño ejemplar del cosmos, incluida la sociedad humana, concebido en la inteligencia divina. ¿Es posible rastrear un camino que nos lleve a una respuesta integradora? En este ensayo queremos indagar, en una primera parte, sobre la búsqueda científica de esa respuesta, y en una segunda parte, desde la luz de la fe, examinar a qué corresponde el Gran Diseño como una aproximación al misterio de la mente de Dios.

\section{El desarrollo del conocimiento científico}

El conocimiento científico actual, con sus abismales logros y limitaciones, solo podemos entenderlo si nos asomamos brevemente a la historia de sus orígenes. En los inicios del conocimiento sistemático, hoy pareciera lo contrario, la filosofía fue la ciencia básica troncal de los demás conocimientos. La frontera entre ambos conocimientos de filosofía y ciencia no requería de una demarcación exacta y definida, porque, con justa razón, consideraban el objeto de estudio como una realidad poliédrica a la cual se acercaban desde diferentes intereses y niveles epistémicos. Esta situación se mantuvo desde los antiguos filósofos griegos y romanos hasta muy entrada la edad moderna con científicos como Bacon, Newton, Kepler, etc. Igualmente Descartes prescindía de hacer la diferencia. Concibe a la filosofía como el gran árbol, cuyo tronco es la física y las ciencias son las ramas. Por esta misma razón, Newton, que escribe el primer trabajo científico en términos modernos, titula su investigación así: Principios Matemáticos de Filosofía Natural (Capra, 1993).

Por su puesto, los antiguos filósofos hacían filosofía y ciencia a la vez. Su interés científico estaba dirigido a conocer las causas últimas y próximas de los fenómenos de la realidad. Esta, según Platón (Fedro, 1979), se da a conocer en forma sensible a través de los sentidos y en forma racional a través de las ideas. Existe un dualismo heredado de Heráclito y Parménides, que Platón no logra superar. El conocimiento del mundo sensible es cambiante y errático, produce solamente opinión (doxa); el conocimiento, en cambio, de las ideas es verdadero y permanente, alcanza la verdad de las cosas (alezeia), y es por tanto científico. Al valor 
de científico se llega mediante el conocimiento de las realidades del mundo superior, que está integrado por ideas eternas e inmateriales. El universo físico es solamente una sombra del verdadero y real mundo de las ideas.

El conocimiento científico en Aristóteles (1973) se distancia del planteamiento platónico. Parte del acercamiento a la realidad de las cosas. Mediante el proceso de abstracción la mente penetra las cosas o los fenómenos, conoce la verdad y puede explicar su estructura y su comportamiento. Así, la física aristotélica reconocía diversidad y jerarquía en los seres del universo, que era el lugar de las cosas y de los seres humanos. Dentro de ese ordenamiento natural los seres se distinguían por el grado de perfeccionamiento de su forma. Todos los seres, de acuerdo con su teoría hilemórfica ${ }^{2}$, están compuestos de

2. El hilemorfismo aristótelico es una teoría fundamental en la concepción de la realidad y del ser humano. El término proviene de dos raíces griegas que significan materia (hile) y forma (morfé). Todo ser material está compuesto de materia y forma. En el caso de los seres vivos la forma es el alma, $y$ en el caso del ser humano la forma es el alma racional. Su planteamiento es una crítica radical a las ideas platónicas y una afirmación de la existencia del mundo real. Esta teoría caló tan profundo que fue base de la filosofía y teología medieval. Pueden consultarse las obras de Edwin Hartman, 1977, Substance, body and soul: Aristotelian investigation, Priceton U.P. y José M. Petit, materia prima y forma sustancial. Así tenemos seres inanimados, seres vivos y seres racionales. En esta escala, los seres humanos, con una forma más completa, serían los seres privilegiados del universo conocido, con un mayor perfeccionamiento, que proviene de la conciencia racional .

El conocimiento científico, estancado y atrapado por el método escolástico durante el medioevo, resurge en el renacimiento y en la edad moderna, ahora impulsado por el método empírico y las matemáticas. Las ciencias de la naturaleza son las primeras beneficiadas con el nuevo método. Este cambio va a dar origen a la revolución copernicana (s. XVI) y a una nueva imagen del universo. De una concepción finita, donde la tierra es el centro del universo, se pasa a una concepción heliocéntrica de un universo infinito en extensión (Kuhn, 1994: 185).

El posicionamiento del método empírico matemático da origen a la cultura de la ilustración y a la entronización de las ciencias positivas en los siglos XVII al XIX principalmente, para las que la validez y certeza del conocimiento está fundado en el conocimiento empíricamente demostrable. Desde esta

y Antonio Prevosti,, 2004, Filosofía de la naturaleza: Su configuración a traves de sus textos, Barcelona: Ed. Scire. 
perspectiva, Comte (1788-1857) establecía tres etapas en el desarrollo del conocimiento: El conocimiento mítico, el conocimiento metafísico, y la etapa del conocimiento científico, que es el grado más alto y perfecto. Sin embargo, el mismo avance de las ciencias y nuevas corrientes de la filosofía contemporánea se han encargado de desmentir tal aserto. Husserl (1859-1938) crea y establece otra vía o método para hacer ciencia: la fenomenología. Se trata de un método riguroso, ordenado y sistemático, abierto a toda la realidad material, humana y social, que trata de llegar a la esencia de las cosas y fenómenos que estudia. Los aportes de Husserl dieron impulso al desarrollo de las ciencias humanas y sociales (psicología, antropología, arqueología, historia, ciencias sociales, filosofía, teología, etc.), contribuyendo a la creación de nuevos métodos, como el histórico, el histórico-crítico, el arqueológico, el hermenéutico, el dialéctico, entre otros.

De esta manera, se pasa de un concepto de ciencia positivista reduccionista validada por la verificación empírica a un concepto de ciencia amplio cuya validez está dada por la rigurosidad del método aplicado. El empiriocriticismo neopositivista y los resultados actuales de las ciencias naturales reconocen que la verificación empírica no es un dato cerrado, sino procesal y complejo, donde intervienen tanto el sujeto como el objeto investigado en el marco de un contexto histórico sociocultural. Desde esta perspectiva, la construcción de la imagen del universo o los distintos modelos creados sobre el origen del universo como el Big Bang ${ }^{3}$ (Gran Explosión), el Big Crunch (Gran Colapso), o el de los universos paralelos, con base en la física matemática, son solamente una cara del poliedro.

\section{El conocimiento primitivo del universo}

Las observaciones y el asombro ante un cielo estrellado y aparentemente inmutable han sido desde tiempos inmemoriales un motivo para preguntarse y tratar de construir una imagen física del universo de acuerdo con el nivel de conocimiento y cultura de cada pueblo. Así, la astronomía y la cosmología han estado unidas a la tendencia a construir explicaciones de

3. La teoría del Big Bang responde a la vieja e inquietante pregunta que el hombre de ciencia se ha hecho sobre el origen del universo. Dice Sagan, en Cosmos, 1997: 20, que un sacerdote babilonio llamado Beroso escribió un libro (perteneció a la Biblioteca de Alejandría), cuyo primer volumen estaba dedicado al período que va de la creación al diluvio con una duración 432.000 años. Hoy la ciencia reconoce que de esa gran explosión a la actualidad hay unos 20 mil millones de años aproximadamente. 
los fenómenos del cielo vinculados a los terrestres.

El inicio de las investigaciones astronómicas se da en el Neolítico, 9000 a 3000 años a. C. Es concurrente con el desarrollo de las grandes civilizaciones de la antigüedad. Carmen Toro y Llaca (1999) identifica 6 grandes culturas hacia el $6000 \mathrm{aC}$, la mayoría de ellas asentadas junto a grandes ríos, que sirvieron de sostén de su economía y de su vida socio-cultural. Estas culturas fueron: La Hassuna, que dará origen a la Sumeria y a la Babilónica, ubicada entre el Tigris y el Eúfrates; la Egipcia junto al Nilo; la Indú, que culmina con la civilización Harappa, junto al Indo; los primitivos asentamientos que son la base de cultura China, junto al río Huang-ho; y la Maya y Azteca en Mesoamérica, y la Nazca e Inca en el Altiplano Andino, cuyo desarrollo es posterior a las euroasiáticas (1500 y 2100 , respectivamente).

Estas culturas, cada una en su contexto histórico, construyeron su propia imagen del mundo. En unos casos fue especulativa y fantasiosa; en otras, en cambio, fue el resultado de repetidas observaciones apoyadas en mediciones matemáticas. Entre las más interesantes en relación con nuestro objetivo está la cosmología protohindú para la que el mundo se apoya sobre cuatro elefantes y estos, a su vez, sobre una tortuga, que flota en el océano universal. En el centro del universo hay siete zonas concéntricas, de las cuales la interior está dividida en cuatro continentes. Uno de ellos es la India. Pero, más allá de esta visión fantástica del mundo, los Sumerios, hacia el año 3000 a $\mathrm{C}$, fueron la primera gran cultura organizada, que desde los Zigurats sistematizaron sus observaciones con el interés de explicar el origen y naturaleza del universo. La cosmología sumeria definió el universo como un globo esférico, inmóvil y en equilibrio, inmerso en un océano cósmico infinito. Por su parte, la tierra es una especie de disco plano, que flota horizontal al mar, que es origen de todas las cosas y que está cubierto por la bóveda del cielo, lugar donde se desplazan los astros: el sol, la luna, los cinco planetas "errantes": Mercurio, Venus, Marte, Júpiter, Saturno, y las estrellas. Agruparon las estrellas en 12 constelaciones para conformar el zodíaco y crearon calendarios lunares de 12 meses de 29 y 30 días.

Hacia el 4000 a. C., otra gran cultura que, similar a la Sumeria, influenció la filosofía y la ciencia griega, la helenística y la medieval, fue la Egipcia. De acuerdo con esta cosmología, la tierra tiene forma de plato alargado, en cuya dimensión mayor corría paralelamente el Nilo. Encima de la tierra estaba el dios aire que sostenía una segunda bandeja en forma de bóveda, que era el 
cielo. Por debajo de la tierra existía una tercera bandeja que contenía al dios de las aguas, sobre las que flotaba la tierra. Afirma Kuhn (1994) que la cosmovisión egipcia obedecía al conocimiento y experiencia que tenía de su país, cuya economía, vida y cultura se movían en función de los cambios estacionales del río Nilo. Las observaciones con menos precisión que las sumerias estaban asociadas a grandes mitos que abarcaban creencias profundas sobre la vida y la muerte. El sol, conocido como Ra, era el principal dios de los egipcios. Utilizaba dos embarcaciones, una para viajar de día a través del aire y otra en su viaje nocturno sobre las aguas. Las estrellas, como dioses menores, estaban claveteadas en la bóveda celeste. Distinguían entre ellas a las estrellas circumpolares, porque no descienden por debajo del horizonte. Por eso, llamaban a los cielos del norte el lugar donde no existe la muerte y donde se goza de una vida eterna y feliz.

Estas primitivas cosmologías son especies de bosquejos de una estructura del universo y responden a necesidades psicológicas profundas como la de sentir al resto de la naturaleza y al universo como su espacio vital donde todas las actividades físicas y espirituales están integradas. Sin embargo, estas cosmologías son simples bosquejos esquemáticos, que solo denotan pero no explican los fenómenos como se le exige a las cosmologías científicas. Las cosmologías, primitivas o no, como visiones de mundo están, por tanto, sujetas a cambios producidos por los avances de la astronomía, que es la ciencia encargada de verificar las observaciones de los fenómenos del cosmos (Kuhn, 1994:29-31). Veamos a continuación algunos aspectos de la primera cosmología científica.

\section{El diseño finito del universo en las cosmologías antigua y medieval}

El aporte griego a la construcción de una visión científica del universo tiene dos momentos. En una primera fase está el aporte de los clásicos con las contribuciones, entre otros, de Tales de Mileto, Platón, Aristóteles (ss. VI - IV a. C.) y en un segundo momento está el aporte helenístico: Eratóstenes, Hiparco, Ptolomeo (ss. III a.C. -II d. C.). La principal característica de esta nueva cosmovisión es el diseño finito y geocéntrico del universo. Aunque diferentes filósofos antiguos y científicos propusieron teorías distintas sobre los fenómenos celestes, el modelo que prosperó con amplia aceptación e influencia en el pensamiento medieval, fue el modelo cosmológico aristotélico tolemaico. 
En qué consistía este modelo finito y geocéntrico? Examinemos seguidamente los aspectos principales de estos dos momentos en forma conjunta:

Aristóteles (384-322 a. C.) tiene la capacidad y el mérito de integrar en su cosmovisión los aportes de la reflexión científica de varios siglos anteriores. Su modelo geocéntrico, asimilado y promovido por los científicos de la Biblioteca de Alejandría, perduró por 22 siglos. Según su teoría el cosmos se divide en dos regiones. La región sublunar y la región supralunar. La primera región comprende 4 elementos: la tierra, agua, aire y fuego. La tierra es una esfera inmóvil y el centro del universo. Aristóteles demostraba que no era plana, sino redonda. Uno de sus argumentos probatorios fáciles de observar era la pérdida de visibilidad de un barco en la medida en que se alejaba del punto de partida. Eratóstenes (280-200 a. C) midió el perímetro de la tierra utilizando la altura del sol de mediodía y el resultado fue 39. $690 \mathrm{kms}$, dato inexacto comparado con el actual; sin embargo, el método utilizado era correcto.

La región supralunar o celeste se extendía desde la Luna hasta las estrellas fijas. Esta región está compuesta de Éter, elemento procedente de la naturaleza divina y en ella se ubicaban siete esferas, que correspondían a: La Luna,
Mercurio, Venus, el Sol, Júpiter, y Saturno. Más allá de Saturno se encontraban las estrellas organizadas en galaxias y constelaciones, trabajo de agrupación que los sumerios iniciaron hace más 3000 años a. C. Las estrellas estaban como fijas en el firmamento, pero tenían un leve movimiento armónico relativo, circular y uniforme. El impulso era dado por un primer motor inmóvil. Todas las estrellas, dentro de sus respectivas constelaciones, se mueven al unísono, pero conservando su posición y su forma. ¿Qué significa todo esto? Una estrella de la Osa Mayor, en el hemisferio norte, o de Aries, siempre conservará la forma y la distancia respecto a las demás (Kuhn ,1994:39). Más allá de la esfera de las estrellas no había nada, ni espacio, ni materia. De ahí el carácter finito de esta imagen cosmológica.

Dentro de esta visión cosmológica el Sol era considerado un planeta en movimiento. Un movimiento aparente de este a oeste durante el día y otro que realiza día a día, durante el año, a través de la eclíptica ${ }^{4}$. Aristarco de Samos (310

\footnotetext{
4. Es una línea curva o trayectoria del sol en su "movimiento aparente sobre la tierra". El término está relacionado con los eclipses del sol y de la luna. Las órbitas de los planetas del sistema solar están próximas a la eclíptica. Si la sombra de la luna se interpone entre la tierra y el solo, se produce un eclipse solar. En cambio, si la sombra de la tierra se in-
} 
- 250 a. C.), en cambio, se distanciaba de la teoría de las siete esferas alrededor de la tierra, y proponía otra en la que el Sol era el centro y los demás planetas, incluyendo la tierra, giraban en torno a él. Esta teoría, retomada en el Renacimiento, no tuvo en su momento la aceptación suficiente.

El nuevo diseño del universo, según los filósofos y científicos griegos, fue un logro de la razón científica . Dejaron de recurrir a los dioses y en su lugar aplicaron métodos científicos para explicar los fenómenos de la naturaleza.

El universo, según el nuevo diseño, tiene forma esférica y circular. ¿Por qué? Dice Platón (1979) en el Timeo que la forma esférica y circular es "la más perfecta de todas las figuras y la más completamente semejante a sí misma. Pues lo semejante es mil veces más bello que lo desemejante". En realidad, el universo está compuesto por dos esferas: una interior, la tierra, en la que se desarrolla la vida humana, y otra externa, celeste, integrada por las estrellas.

Ptolomeo (87 - 170 d. C.) recoge en su obra maestra, Syntaxis

terpone entre la luna y el sol, tendremos un eclipse lunar. Este comportamiento fue medido, con cierta exactitud, por los científicos de Alejandría 200 años a C. Sobre el tema véase "El antiguo universo de las dos esferas" en Kuhn, La Revolución copernicana. matemática, posteriormente conocida como Almagesto, todo el avance de la astronomía y cosmología de los clásicos griegos, en especial, la cosmología aristotélica. Intenta explicar matemáticamente la estructura del universo y las distancias entre los planetas tomando como centro a la Tierra. Trata de resolver el problema de Platón o el retroceso aparente de los planetas señalando que hay un punto denominado Ecuante ${ }^{5}$, que está situado a la misma distancia del deferente con la tierra y que elimina el problema. Asimismo, resuelve el problema de la diferencia de brillo de las estrellas, la cual se da según la distancia entre el planeta y el Ecuante. Otra labor de Ptolomeo fue la clasificación de 1022 estrellas en función del brillo aparente. Afirma Kuhn que la compleja combinación de excéntricas, epiciclos y ecuantes aportadas por Ptolomeo no lograron conciliar la teoría con la observación en forma precisa, de modo que el sistema propuesto no fue ni el más complejo, ni una

5. Es un punto cercano al centro de la esfera del planeta, en el marco de la teoría geocéntrica. Esta teoría estuvo vigente desde el s. VI a C. hasta el s. XVI en que fue sustituida por la teoría heliocéntrica. Ptolomeo utiliza el ecuante para mantener la teoría de Platón del movimiento circular uniforme de los cuerpos celestes. Por su parte, la deferente es un círculo que tiene por centro a la tierra. 
versión científica última y definitiva (1994:110).

Aunque hubo otros modelos del cosmos, entre los griegos el modelo científico aristotélico ptolemaico fue el más completo y aceptado, sin discusión crítica, en el mundo cultural del imperio romano y el medioevo. En primer lugar, el sistema numérico introducido por los romanos no favorecía el avance de las ciencias y el cálculo matemático y, en segundo lugar, la desmoralización de la sociedad del imperio, como señala S. Agustín (354-430) en su obra Civitas Dei, fue la polilla que carcomió las bases políticas, militares y culturales del imperio, lo que significó la destrucción del imperio de occidente en el año 476 y el estancamiento de las ciencias y técnicas hasta que tomaron un nuevo empuje en el Renacimiento. Desde otro ángulo, la filosofía y teología cristiana medieval, en los primeros siglos tuvo una gran influencia platónica, y a partir del siglo XII, con Tomás de Aquino (1225-1274), la influencia fue aristotélica. En consecuencia, la cosmovisión cristiana medieval se asentó en el diseño científico de Aristóteles y de Ptolomeo. Una síntesis de esta cosmovisión la encontramos bellamente descrita en la obra literaria de Dante Alighieri (1265-1321), La divina comedia.

Sin embargo, desde el punto de vista teológico, existían algunas contradicciones en la cosmología aristotélica. Una de ellas era la eternidad del universo, que se oponía a una verdad fundamental del cristianismo: "En el principio Dios creó los cielos y la tierra" (Gén. 1, 1-2). ¿Qué significa “crear" en el contexto de la Biblia? Sobre este pasaje haremos su análisis más adelante.

A diferencia del atraso científico que padecía Europa medieval, el mundo y civilización árabe, a partir del siglo VI, dio muestras de crecimiento progresivo en el orden económico, político y cultural. Bagdad fue la nueva capital del desarrollo de las ciencias árabes y al-Mansur (754-775), el califa responsable de impulsar la traducción de numerosas obras de astronomía indú, persa y griega. En el siglo IX Isha Ibn Hunayn tradujo la obra de Ptolomeo Syntaxis matemática dándole el nombre de Almagesto. Siglos más tarde Averroes (1120-1198), perteneciente al mundo árabe hispánico, es considerado el científico de mayor influencia en la cultura occidental. Su principal obra es " $\mathrm{Co}$ mentarios a la obra de Aristóteles", comentarios que sirvieron para dar a conocer el pensamiento aristotélico entre los filósofos y teólogos medievales de la época. Si bien es cierto que la cosmovisión aristotélica era ampliamente reconocida y base del universo cristiano, esta encerraba imprecisiones y contradicciones. 
Algunos científicos medievales y precursores de Copérnico se encargaron de criticarlo y formular aclaraciones. Tal fue el caso de Nicolás de Oresme (1323-1382) y de Jean Buridan (1300-1358).

\section{El diseño moderno del universo según las leyes positivas de la física teórica}

La base del diseño actual del universo es una asociación de conocimientos provenientes de las ciencias: termodinámica, hidrodinámica, astronomía, física atómica, física cuántica y relatividad, entre otras. ¿Qué es la física teórica? Es una ciencia positiva interdisciplinar que intenta comprender, explicar y predecir los fenómenos de la naturaleza a partir de un modelo matemático y conceptual. Su núcleo central es la matemática y su método es experimental o empírico descriptivo.

Los logros de estas ciencias modernas y contemporáneas han roto los límites del espacio y del tiempo y empujan la frontera del conocimiento del universo hacia mundos desconocidos. Nuevos estudios y mediciones revelan que la estructura del cosmos ha cambiado en relación con el modelo aristotélico ptolemaico. El Sol, desde el nuevo paradigma de Copérnico (1473- 1543) dado a conocer en su famosa obra De revolutionibus orbium caelestium, es el centro del universo y la Tierra un planeta con movimiento circular alrededor del Sol. A esta primera ruptura paradigmática que provocó la revolución copernicana se han sumado los aportes de Galileo, Kepler, Newton, y de contemporáneos como Friedman, Gamow, Lemaitre, Hubble, Hawking, etc., con resultados como los siguientes: El diámetro de la tierra asciende a 12.756 kilómetros; la luna está alejada de la tierra 30 veces esta distancia o sea 384.400 kilómetros y el sol se encuentra a 150 millones de kilómetros. El cielo considerado fijo y limitado en número de estrella, ahora en la nueva visión contemporánea, está poblado por 500.000 millones de galaxias, a distancias que se miden en años luz. Alfa de Centauro, que es la estrella más cercana al sol, se encuentra a 4,36 años luz de la tierra y cada año luz comprende 9.461.000.000.000 kilómetros. Las galaxias son conglomerados de estrellas en la bóveda del cielo. Una de ellas, la más conocida desde la antigüedad, es la Vía Láctea, a la que pertenece el sol. Contiene aproximadamente 200 mil millones de estrellas y un diámetro de 80.000 años luz. Una de las más grandes y brillantes es la galaxia Andrómeda, que se encuentra a 2.5 millones de años luz y una de las más alejadas, la A 1689-z D1, asequible por el telescopio de 
mayor alcance, el Hubble, está situada a 240.000 millones de años luz aproximadamente (Weizsacker, 1974: 138-140; Universia, 2008).

Por una parte, es evidente que el diseño antiguo ha cambiado. Ahora nos encontramos ante un universo cuya extensión es casi infinita, donde la tierra es, según Sagan (1934-1996), un punto físicamente insignificante. Cada vez que el poder de captación de los telescopios aumenta, las fronteras del espacio y del tiempo retroceden. Algunos científicos plantean la hipótesis de la existencia de millones de otros universos mayores o menores al nuestro, que podrían ser una especie de "huevos cósmicos" en un gigantesco nido. Por otra parte, frente a un universo de estrellas fijas con pequeños movimientos circulares uniformes, que participaban de la inmutabilidad y eternidad divina según el diseño aristotélico, ahora en el diseño de la física teórica y de científicos actuales el universo, como un todo, se mueve y se expande. Continuamente mueren y nacen nuevas estrellas. Por ejemplo, las nebulosas son restos o polvo de estrellas, que llegaron a su final y colapsaron. Así, las enanas blancas o las supernovas son originadas por gigantescas explosiones estelares, lo que da una idea impresionante de la expansión del cosmos.
La teoría de la expansión del universo o teoría del Big Bang es la más conocida en la actualidad. El padre de esta teoría es Georges Lemaitre, quien en 1929, después de desarrollar las ecuaciones de la relatividad de Einstein sobre la expansión del universo, propuso que el universo se inició con la explosión de un átomo primigenio. Uno de los científicos que continuaron trabajando esta teoría y que la ha popularizado con sus publicaciones es Stephen Hawking. Sobre el tema ha publicado Breve historia del tiempo, El universo en una cáscara de nuez, Teoría del todo, La naturaleza del espacio y del tiempo, y la más reciente: The grand design.

En estas obras Hawking se plantea preguntas, que desde el neolítico el hombre se ha venido haciendo al observar, contemplar, y medir matemáticamente el universo, y también plantea respuestas, algunas muy aventuradas, que van más allá del campo especializado de la física teórica actual. Entre otras preguntas sobresalen las siguientes: ¿Tuvo un origen el universo o existe desde la eternidad? $\mathrm{Si}$ tuvo un inicio, ¿es Dios el creador o el mismo se autocrea? Alrededor de estas preguntas y en contexto semejante, Sagan (1984), además, se preguntaba: ¿Qué o quién es Dios? Y afirmaba: la respuesta depende mucho de qué se entiende por ese término. Los diferentes significados 
han dado origen a todas las religiones, desde las monoteístas a las naturalistas, que han existido a lo largo de la historia de la humanidad. Para algunos, continúa, Dios es un hombre de piel blanca y de inmensos poderes, en cambio, para otros como Baruch Spinoza o Einstein, Dios es la suma total de las leyes físicas.

Es notorio que el avance de las ciencias matemáticas ha llevado el debate sobre el origen y eternidad del universo a posiciones apresuradas y contrarias a la esencia misma de la ciencia de parte de algunos científicos de la naturaleza. Interpretando estas creencias, Sagan afirmaba:

"A medida que vamos comprendiendo el universo, van quedando menos cosas para Dios. ... Si en Grecia se hubiese inventado, en el siglo $V$ a. J. C., el cálculo diferencial e integral o la aritmética transfinita, y no hubiesen sido desestimadas posteriormente, la historia de la religión en Occidente hubiese podido ser muy distinta - o, por lo menos, se hubiese dado con menor frecuencia la pretensión de que la doctrina teológica puede demostrarse convincentemente mediante argumentos racionales a aquellos que rechazan la revelación divina como intentó Tomás de Aquino en su Summa contra Gentiles. ... Cuando Newton explicó el movimiento de los planetas recurriendo a la teoría de la gravitación universal, dejo de necesitarse que los ángeles empujasen los planetas. ... Se dice que Laplace presentó una edición de su trabajo matemático Mecanique celeste a Napoleón, ... quien se quejó a Laplace de que en el texto no apareciese ninguna referencia a Dios. La respuesta de la Place fue: Señor, no necesito esa hipótesis" (1984:374-376).

Aunque se puede pasar del Dios como hipótesis de Laplace al completo exilio de Dios de la creación del universo como en $E l$ Gran Diseño de Hawking, ya que es perfectamente posible demostrar que el Big Bang fue el inicio del universo y que igualmente son posibles infinitas creaciones y destrucciones del mismo, Sagan reconoce que estamos frente a dos profundos misterios y que nada hace evidente que uno esté mejor posicionado que el otro. Por tanto, lo menos que se podría esperar, sobre todo sabiendo lo que somos a nivel cósmico y los conocimientos aún raquíticos que poseemos, es una actitud humilde y prudente de búsqueda de la verdad. La certeza en las ciencias es relativa, no absoluta. La arrogancia intelectual positivista no llega muy lejos, como lo ha confirmado la epistemología actual. Los logros científicos pueden generar dos actitudes opuestas: Una, la del espíritu arrogante que trata de imponer su verdad como la verdad, y la del espíritu humilde 
que, por el contrario, trata de integrar verdades y soluciones. Así, sin arrogancia, el diseño aristotélico tolemaico integró visiones de la teología, de la filosofía y de las ciencias y funcionó por 22 siglos. Por eso, para Sagan ciencia y teología, si bien parten de principios diferentes, participan de objetivos semejantes, no excluyentes sino complementarios. Sin embargo, Sagan, pese a su gran honestidad y claridad intelectual como se hace notar especialmente en su escrito En el "Valle de las sombras", no logra captar que esos objetivos semejantes tienen alcances también diferentes (1984:378). A continuación, analizamos estos alcances.

\section{La teología, su método y el gran diseño de Dios}

Como las ciencias de la naturaleza y la teología tienen objeto, objetivos, presupuestos teóricos $\mathrm{y}$ contenidos diferentes, los alcances de la teología son más que formales y llegan a lo más profundo de la vida y de la existencia humana.

A diferencia de las ciencias formales y factuales que están creando, como parte de su propia naturaleza y método, nuevos diseños o modelos del universo o de sus respectivos objetos de estudio, la teología (1) y su método avanza en la reflexión y en la interpretación de un solo diseño o modelo que
Dios ha revelado al hombre y que contiene la historia de la salvación. ¿Qué es la teología como ciencia o cuál es su naturaleza y su método?

Dentro de la clasificación anterior de las ciencias la teología es una ciencia factual especial porque su paradigma o sistema de creencias parte de la razón y de la fe. Posee o versa sobre un objeto que es Dios o la palabra revelada de Dios. Esa palabra es una palabra viva, actual, que dialoga con cada lector u oyente de todas las edades y lugares. Como ciencia humana es sistemática y rigurosa. Para este propósito utiliza uno o varios métodos como el método teológico, el método hermenéutico, el arqueológico, y el histórico crítico, entre otros.

La teología reflexiona, estudia e interpreta los textos revelados a la luz de la fe. Razón y fe son imprescindibles en el estudio teológico. Desde el inicio de la edad moderna Melchor Cano (15091560) propone en su obra De locis theologicis una sistematización del trabajo teológico. Identifica y propone los lugares teológicos en los que la investigación teológica debe fundamentarse en la búsqueda de razones y argumentos para escuchar, interpretar, y explicar el dato revelado. El se refiere a diez lugares, de ellos mencionamos los principales: Las sagradas escrituras, la tradición apostólica, los sínodos y 
concilios, el magisterio de la Iglesia, la fe del cuerpo universal de los creyentes. A estos se debe agregar otras fuentes de importancia, aportados por la investigación teológica contemporánea, como el testimonio de la liturgia, el estudio de la vida de los santos, y los Documentos de las Conferencias regionales, del CELAM, en el caso de América Latina, etc.(Wicks, 2001: 21-23).

Parte del rigor del método teológico está en hacer uso no solo de una fuente o lugar teológico, sino de varias, de modo que, por una parte, se pueda captar las diferentes maneras en que Dios se revela a los hombres y, por otra, se busque dar consistencia y objetividad al análisis realizado. En este mismo sentido, el teólogo debe hacer uso de distintos métodos según lo requiera el objeto particular de estudio. Siendo las sagradas escrituras una fuente principal, como lo señala la Constitución del Vaticano II, Dei Verbum, el método hermenéutico es casi de práctica obligatoria. La Biblia es el conjunto de libros sagrados o revelados por Dios. Contiene el Antiguo y Nuevo Testamento. Fue escrita entre los siglos X a. C. y I d. C. en tres lenguas principalmente: Hebreo, Arameo y Griego, en contextos sociales y culturales diferentes. Por tanto, el teólogo es un hermeneuta que estudia, esclarece e interpreta el texto, los intertextos y los contextos sociales, culturales, lo mismo que los diferentes géneros y estilos literarios, a la luz de la fe. En este sentido, a diferencia del científico positivista para quien los resultados tienen la evidencia de la verificación empírica, el teólogo recorre a tientas el camino de la búsqueda de la verdad sabiendo que navega en aguas profundas y llenas de misterio y que sus resultados solo pueden ser aproximaciones a la inescrutable e inagotable palabra de Dios (Wicks, 2001, Juan Pablo II, 1998). Desde este marco de referencia metodológico analicemos, a continuación, el diseño del universo en el Génesis.

\section{El diseño cosmológico del Génesis}

El término "Génesis" viene del hebreo "Bereshith", que significa "origen del mundo" o "en el principio". Fue escrito por varios autores religiosos, en la versión que conocemos, hacia el siglo VI y V a. C., en el contexto del cautiverio en Babilonia. Este dato es importante para reconocer la influencia cosmológica sumeria en la elaboración del discurso o del texto sagrado. Los exégetas identifican, por lo menos, tres fuentes principales: $\mathrm{La}$ fuente J, Yavista, la fuente E, Eloísta, y la fuente P, Sacerdotal, las cuales están basadas en tradiciones orales del I y II milenio a. C. 
El Génesis es el primer libro canónico de los 46 del Antiguo Testamento. No es un libro científico acerca del origen del universo. Es un libro religioso, que ofrece el comienzo de la historia de la salvación. La estructura de dicho libro contempla tres partes:

a. Cap. 1 al 11: Relato de la creación

b. Cap. 12 al 37.1: Los patriarcas o la elección y formación del pueblo de Dios, y

c. Cap. 37.2 al 50: Historia de José en Egipto: Drama y salvación.

En relación con la primera parte, encontramos dos relatos sobre la creación del universo. El primero comienza:

“En el principio, cuando Dios creó los cielos y la tierra, todo era confusión y no había nada en la tierra. Las tinieblas cubrían los abismos mientras el espiritu de Dios aleteaba sobre la superficie de las aguas ..." (Gen. 1,1-2,4a)

El segundo relato es menos detallado sobre el proceso de la creación y se centra en la creación de Adán y Eva, como figuras protagónicas del comienzo de la historia de la relación de Yavé Dios con el pueblo escogido, Israel. El texto dice así:
"El día en que Yavé Dios hizo la tierra y los cielos no había sobre la tierra arbusto alguno, ni había brotado aún ninguna planta silvestre, pues Yavé Dios no habia hecho llover todavia sobre la tierra, y tampoco había hombre que cultivara el suelo e hiciera subir el agua para regar toda la superficie del suelo.

Entonces Yavé Dios formó al hombre con polvo de la tierra... Dijo Yavé Dios: "No es bueno que el hombre esté solo. Le daré, pues, un ser semejante a él para que lo ayude" ... (Gen. 2, 4b-25),

¿Estamos frente a dos diseños cosmológicos diferentes que responden a pueblos y culturas diferentes? El análisis científico bíblico actual, como un dato esencial, relaciona estos relatos de la creación con la prehistoria y la fe de Israel. En segundo lugar, la diferencia entre ambas versiones se debe a que provienen de dos fuentes distintas: La primera proviene de la fuente sacerdotal (conocida como P) y la segunda, de la fuente yavista (conocida como J). De ahí las semejanzas y las diferencias. La primera es conceptualmente precisa. Enfatiza el tiempo, cada día de la semana, de las acciones de Dios. La segunda enfatiza el espacio y se fija en los detalles: La tierra, las plantas, la manera como formó a Adán y a Eva, el jardín del Edén, en donde Dios coloca a la primera pareja de la 
humanidad, etc. El primero es más abstracto: "El hombre fue hecho a imagen y semejanza de Dios", en cambio, el segundo es más poético y figurativo: "Tomó polvo de la tierra para formar al hombre y luego sopló sobre él". Por otra parte, ambos relatos cosmológicos expresan el comienzo del Pueblo de Israel, se dirigen a la creación del hombre, que es la cúspide en importancia y dignidad de toda la creación, y adquieren unidad y cohesión en la voluntad creadora y personal de Dios, a diferencia de las cosmologías primitivas que se centran en un determinado elemento (von Rad. 1982: 189-190).

La fuente sacerdotal (P), como ya hemos indicado, es conceptualmente más precisa y pretende acentuar la diferencia entre el relato cosmológico israelita y otras cosmologías existentes en los pueblos vecinos. Por eso, utiliza el verbo "crear" en lugar del "hacer" o de "organizar" utilizado en el mito sumerio del origen del mundo. En este mito existen dos principios o sustancias primordiales de la que están hechas las divinidades y todas las cosas. Las divinidades hacen y organizan las cosas que hay en el agua, en la tierra, en el aire y en el cielo. En cambio, "crear", en hebreo bara, significa sacar de la nada o traer a la existencia. Antes de la creación sólo existe Dios y las cosas vienen a la existencia en el momento en que Dios ordena. Consciente de esta diferencia el profeta Baruc (6,58-69) anteponía el poder del Dios de Israel frente a los falsos dioses o ídolos. Y les recordaba a los israelitas en Babilonia no temer a esos dioses falsos y poner toda su confianza en Yahvé Dios. Por otra parte, y más cercano a nosotros, el evangelio de Juan, refiriéndose a este momento trascendental, señala: "En el principio solo existía la Palabra y la Palabra era Dios, quien hizo todas las cosas" (Jn 1.1-3).

Otro dato importante es que el diseño cosmológico del Génesis fue escrito por los autores sagrados hacia el siglo VI a. C., posterior a los grandes acontecimientos salvíficos de la historia de Israel. Se da primero una experiencia personal, fundante y espiritual: Dios que elige a su pueblo de Israel y Dios que libera a su pueblo de la opresión de Egipto (El libro del Éxodo fue escrito entre 1440 y 1400 a. C.). Posteriormente, el autor o autores sagrados reflexionan sobre su entorno, sobre el cosmos o el universo, como la gran casa del hombre, y se preguntan por el creador de todas esas maravillas naturales y su respuesta está dada en la primera parte del Génesis. Así las cosas, examinemos a continuación la relación de la cosmología del Génesis con la historia de la salvación. 


\section{La creación del universo en la historia de la salvación}

La fe de Israel nace de una experiencia espiritual, que consiste en un encuentro personal de Yahvé Dios y Abraham, en una elección y en una promesa: "Serás padre de una gran nación y te bendeciré ..." (Gen 12,1-4). Esta experiencia es personal: Abraham escucha el llamado de Dios; es histórica: Se da en un tiempo y en un contexto determinado: Ur de Caldea, Jarán, Siquem; es profunda y radical: "Deja a tu país, a los de tu raza y a la familia de tu padre". Este es el comienzo de una historia de amistad y alianza entre Dios e Israel, amistad que se prolongará con Isaac, Jacob, y su descendencia, y será probada repetidamente a través de los actos de liberación y de salvación que marcan la historia de este pueblo.

La creación del universo no formó parte de la primera fe israelita, como lo han demostrado los estudios históricos y exegéticos de los libros del Antiguo Testamento. Así, el primer credo que los israelitas repetían y trasmitían a sus descendientes no incluía ninguno de los relatos de la creación, como se puede ver en el texto siguiente:

"Mi padre era un arameo errante, que bajó a Egipto y fue a refugiarse allí, siendo pocos aún; pero en ese país se hizo una nación grande y poderosa. Los egipcios nos maltrataron, nos oprimieron y nos impusieron dura servidumbre.

Llamamos pues a Yavé, Dios de nuestros padres, y Yavé nos escuchó, vio nuestra humillación, nuestros duros trabajos y nuestra opresión. Yavé nos sacó de Egipto con mano firme, demostrando su poder con señales y milagros que sembraron el terror. Y nos trajo aqui para darnos esta tierra que mana leche y miel. Y ahora vengo a ofrecer los primeros frutos de la tierra que tú, Yavé, me has dado" (Dt. 26, 5-10).

En este texto hay tres intervenciones fundamentales de Dios que resumen la experiencia de fe de las tribus primitivas de Israel: "Mi padre, un arameo errante (el nombre de Abraham no es mencionado) que baja a Egipto", "la liberación con mano firme de parte de Dios", y "la entrega de la tierra que mana leche y miel". Es evidente que en este período la creación del universo no es aún considerada como una de las intervenciones fundamentales. Las generaciones posteriores recordarán estos hechos arriba mencionados como grandes manifestaciones sagradas o teofanías, en las que Dios se va mostrando, conduce y cuida a este pueblo elegido. La historia de esta primera fe israelita es una historia de elección y de salvación (1: Jos 24, 2-13; Dt. 6,20-24). ¿Cómo llegan estas 
tribus a tomar conciencia de que la creación del universo es una intervención esencial en la historia de la salvación?

El cautiverio en Babilonia, en el siglo VI a. C., es una nueva experiencia humana espiritual que vive el pueblo de Israel. Lejos de su patria, de sus tradiciones, e inmersos en una cultura ajena a sus principios y creencias los israelitas consideran como una amenaza a su identidad, como pueblo elegido, las nuevas costumbres, la nueva moral y los nuevos dioses de Babilonia. Surgen así los profetas del exilio: Isaías, Jeremías, Ezequiel, etc. Frente a la crisis y el peligro de perder la fe en Yavé, ellos ven la necesidad de anteponer la fe y la esperanza en la restauración de Israel. El centro del mensaje es: "Si Dios es todopoderoso, creador de cielo y tierra, también tiene poder para liberarnos". Por eso, en Isaías 40 ss se afirma que Yahvé ha perdonado los pecados de Jerusalem, y a continuación el profeta repite sin cansancio "la grandeza de Yavé Creador":

"Pongan la cara hacia arriba y miren: Quién ha creado todos esos astros?

El, el mismo, que hace salir en orden su ejército, y que llama a cada estrella por su nombre.
Su fuerza es tan grande y su poder tan inmenso, que ninguna se hace la desentendida. ... ¿Acaso no lo sabes o no lo has oído? Yavé es un Dios eterno que ha creado hasta los extremos del mundo"...

En este contexto profético la tradición sacerdotal redacta la única cosmogonía que hay en toda la Biblia. ¿Cuál es la intención? Los profetas, como también la fuente sacerdotal, pretenden luchar contra las posibles influencias del medio social y cultural del exilio babilónico y de otros pueblos que rodean a Israel. Lo particular y significativo de la reflexión sacerdotal es que interpreta el comienzo del universo en función de la elección y alianza de Dios con Israel. Por eso afirma von Rad (1982) que la bondad de Dios para con su pueblo prima sobre la omnipotencia.

A partir de este período el tema de la creación del universo va a ser una constante en la literatura bíblica. En los Salmos el tema de la creación se convierte en un canto de alabanza: Salm. 19,2 "Los cielos cantan la gloria de Dios". En la literatura sapiencial, bajo influencia helenística, se manifiesta una relación entre la creación y el orden moral: Sab. 11,10: "Todo lo dispusiste con medida, número y peso"; Sab. 13,1: “... Todos los hombres que ignoraron a Dios, .....contemplando su obra, no reconocieron al Artífice". En la etapa final del 
Antiguo Testamento, en el siglo II a. C., en el II libro de los Macabeos 1,24-29 se afirma de manera clara: "Dios creó de la nada todo el universo, es justo y lleno de misericordia". ...Y "es motivo de esperanza para los que mueren" (1,38-43).

En el Nuevo Testamento el tema de la creación continúa como un elemento central en la historia de la salvación, solamente que ahora adquiere un carácter cristocéntrico. Cristo es la plenitud de los tiempos, el Alfa y Omega de toda la creación. Es el pleroma ${ }^{6}$, en el que se cumplen todas las promesas y plenitud de todas las bendiciones (Rm 11, 12.25; Mc 1,15; Rm 15,19). El diseño de la creación solo tiene sentido en Cristo. Por eso Pablo en Rom. 8, 21ss afirma:

“...el mundo creado también dejará de trabajar para el polvo, y compartirá la libertad y la gloria de los hijos de Dios. Vemos que la creación entera gime y sufre dolores de parto. ... en la esperanza de la redención de nuestro cuerpo".

6. Es un término que proviene del griego "pleroo" que significa llenar. Si bien tiene ascendencia gnóstica, es utilizado repetidas veces en las cartas paulinas. Del sentido de plenitud se deriva que todo tiene sentido en Cristo. Por eso, Pablo afirma en Ef. 1,10. 22,29: Cristo es la cabeza del cosmos y por tanto resumen del proyecto divino de salvación. Veáse Pierre Benoit, Revue Biblique 63 (1956) 5-44: Pierre T. du Chardin, El fenómeno humano, Energía humana e Himno al universo.
En consecuencia, la historia de la salvación es una historia de fe. Es un proyecto unitario salvífico, que va desde el comienzo de la creación hasta el final de los tiempos. Sobre este gran proyecto, ¿es posible la alianza y el diálogo entre la fe cristiana y la ciencia?

\section{El Gran Diseño de Dios: fe y ciencia}

¿Qué es un diseño? En el campo de la investigación científica el diseño es un modelo teórico que sostiene y sustenta el desarrollo de la investigación y el objeto de estudio. En relación con la naturaleza, la ciencia y, en especial, la física teórica construyen un modelo y un método para estudiar el ser y el comportamiento de la realidad. Si el modelo es correcto y efectivo podrá descubrir e identificar un modelo implícito de leyes o normas que rigen la estructura interna y el comportamiento del ser o de la realidad, y por tanto podrá predecir consecuencias y resultados verificables de ese comportamiento. Las leyes o normas identificadas conforman un diseño esencial, que lo poseen desde las partículas elementales más pequeñas (subatómicas) hasta la galaxia más grande y más alejada del universo. Con esto, estamos diciendo que el universo físico tiene y obedece a un diseño y de acuerdo con ese diseño, descubierto 
por la ciencia, tuvo uno o muchos comienzos lo mismo que podrá tener uno o muchos finales.

En la época medieval el concepto de "diseño de la creación del universo" adquiere contenidos filosófico-teológicos orientados a fundamentar la racionalidad de la fe. Tomás de Aquino (1225-1274) recoge de las sagradas escrituras y de los padres de la Iglesia los elementos básicos necesarios para plantear la tesis de la causalidad ejemplar de Dios, especialmente en dos de sus obras principales: $D e$ veritate y Summa Theologica. En la primera se afirma que el universo no es producto de la casualidad, sino que responde a una idea o a un "diseño", concebido en la mente de Dios. Esto significa que Dios es el artífice inteligente o la causa ejemplar del universo (De ver. q. 3). En la segunda, continua afirmando: "El mundo y cada cosa que lo compone tienen en Dios su idea ejemplar". El mundo se hizo y se está haciendo según un plan preexistente en la mente divina (Sum. Th. 1,15-23; 1, q.14-16). Es decir, el modelo implícito de leyes que norman el ser y el comportamiento del universo está en la mente divina. ¿Qué es la mente de Dios? Es la Sabiduría de Dios. ¿Cómo entrar o llegar a ella? En Proverbios 8, 22 ss. se indica una vía: Dios es el artífice que ha hecho todas las cosas siguiendo un plan. Ese plan es anterior al cielo, a la tierra y cuanto existe, y es lo que le da armonía a todo el universo, incluido el hombre y la sociedad. Pero no basta el conocimiento o la ciencia de las cosas. El capítulo termina con un mensaje moral religioso: la felicidad consiste en seguir sus caminos, los caminos de este plan; encontrarlo y aceptarlo significa encontrar la vida y, por el contrario, despreciarlo a conciencia es elegir la muerte. Entonces, de acuerdo con el autor sagrado, solamente se llega a él o a la Sabiduría a través de la fe, que es la aceptación de sus enseñanzas.

Así, el Gran Diseño no consiste solo en un modelo o teorías del universo físico, aunque tengan el mayor reconocimiento de la astronomía moderna o de la física teórica contemporánea. Para un creyente cristiano actual el Gran Diseño es algo más complejo e integral, es la historia de la salvación, fundamentada en una concepción lineal de la historia y del tiempo. Dios está al inicio, creando, manteniendo en su ser a todos los seres creados y guiando toda la creación hacia el final de la historia y del tiempo. ¿Qué sentido tendría el "gran nido cósmico", la vida y la historia de la sociedad humana separado de la historia de la salvación del hombre? Todo acabaría absurdamente con la muerte de las personas, la vida maravillosa del planeta, y todo el sistema cósmico. 
De ahí que para Tomás de Aquino y para la teología católica, Dios no solo es la causa ejemplar, sino también la causa final de toda la creación. La dimensión de la fe permite dar sentido a los miles de millones de años de evolución cósmica y al desarrollo de la vida neuronal y espiritual del ser humano.

Fe y ciencia son dos fuentes de conocimiento que se complementan. No pueden contradecirse si ambas buscan la verdad mediante caminos legítimos y diferentes. Por eso, Rahner, en relación con esa fuerte vinculación, afirma que la historia de la salvación acontece en la historia humana, la abarca y la integra a una plenitud mayor, y Juan Pablo II en Fides y Ratio (1998) apoyado en las fuentes bíblicas señala : Hay una profunda unidad entre el conocimiento de la razón y el de la fe (p. 29). Advierte que el hombre, también el hombre de ciencia, con solo la luz de la razón puede encontrar y reconocer el camino hacia Dios, pero sólo podrá recorrerlo desde el horizonte de la fe (pp. 30, 33 ).

Sin fe el hombre corre el riesgo de quedarse en el camino paladeando la felicidad que ofrecen las delicias terrestres, sabiendo y aceptando que al final solo está la muerte (Camus, 1939), o como en el caso de Sagan que enfrentado a la muerte le basta "entrever la maravillosa estructura del mundo existente, junto al piadoso esfuerzo por comprender una porción, aunque sea diminuta, de la razón que se manifiesta en la naturaleza" (1998: 288).

Sagan, como muchos otros científicos, llegan al umbral del misterio, pero no dan el paso hacia la gran aventura de la fe. Entrar en el misterio significa encontrar a Dios, pero este paso trascendental está mediado por la libertad soberana del hombre. Entrar es un acto valiente que implica, por una parte, despojarse de todas las certezas y seguridades materiales y humanas, como en el caso de Abraham, de Moisés, Pablo de Tarso, y, por otra, aceptar dócilmente ser conducido para iniciar un recorrido de luces y de sombras, de paradojas, como dice Karl Barth en su Comentario a la Carta a los Romanos, pero seguro de que Dios, el Dios de la historia de la salvación, la fuerza del bien, está a tu lado y te sostiene. Tampoco es correcto ni cierto afirmar que la fe esté asociada a "debilidades de la mente" o al "miedo a la muerte". La sagrada escritura y la historia de la Iglesia dan testimonio de lo contrario, es decir, que la fe es una experiencia personal, íntima y profunda que abarca todo género de personas, cuyas creencias los lleva a vivir y a realizar acciones heroicas o sencillas, pero que dan muestras de ser gigantes en el conocimiento y en la vivencia de su fe. 
En la Iglesia Católica, desde sus inicios, ha habido un permanente esfuerzo, apertura y trabajo por integrar conocimiento y fe, ciencia y teología, sin perder de vista la especificidad de cada una. En el último siglo es bueno recordar algunos ejemplos, como el de Georges Lemaitre, teólogo y astrofísico belga, descubridor de la explosión del átomo primigenio (1931), conocido ahora como Big Bang; Pierre Teilhard du Chardin (1881-1955), teólogo, filósofo y paleontólogo, defensor de la tesis de una evolución cósmica, que culmina en el punto Omega que es Cristo; y Leonardo Boff, teólogo y ecologista, para quien "más allá del vacío cuántico, la última referencia de la razón analítica, está el océano de energía, el continente de todos los posibles contenidos, el "gran atractor cósmico", pues se percibe que el conjunto del universo está siendo atraído hacia un punto central misterioso" (2003:79). En esta cosmovisión teísta Boff trata de integrar los nuevos paradigmas de las ciencias. No menos significativo es el testimonio de Francis Collins, 2008, genetista y Director del proyecto del Genoma Humano, para quien existe una maravillosa armonía entre las verdades complementarias de la ciencia y la fe. El Dios de la Biblia, sigue afirmando, es el mismo Dios del Genoma y la ciencia puede ser en efecto un medio para encontrar y aceptar a Dios, que es el artífice del Gran Diseño de la historia de la salvación.

\section{Conclusión}

La obra de Hawking, The Grand Design, ha sido el motivador de estas reflexiones cosmo-teológicas. Hablar del cosmos es hablar de la existencia de miles de millones de estrellas, galaxias y planetas, expandiéndose a distancias asombrosas, donde la tierra y el hombre son insignificantes dentro de la escala cósmica. Del neolítico a nuestros días el avance del conocimiento científico tecnológico, en particular los logros de las ciencias de la naturaleza, han permitido plantear la hipótesis del "átomo primigenio" cuya explosión poderosísima o Big Bang sería el origen del universo y de todo lo que él contiene.

Algunos científicos del pasado y del presente, como Hawking actualmente, impresionados por estas conquistas de la ciencia, afirman: "el maravilloso diseño del universo no tuvo diseñador". En este trabajo hemos querido acercarnos a un concepto de ciencia más amplio, más abarcador e integrador donde los aportes de la física teórica no son verdades absolutas, sino simples hipótesis de trabajo, parciales con respecto a una realidad poliédrica y densa en significados, que lejos de aportar una solución 
definitiva, lo más que hacen es darnos, como dice Sagan, una cierta madurez para abrir nuevos caminos de búsqueda.

Motivado por los avances de las ciencias humanas y por el método fenomenológico e histórico crítico nos hemos acercado a la visión judeo cristiana de la creación del cosmos. Para esta cosmología teológica no hay diseño sin diseñador. El universo no tiene sentido en sí mismo. Dios, el señor del espacio, de la gravedad y del tiempo. La visión paulina de esta cosmología es total e integradora. Todo tiene sentido en relación con el hombre y el hombre encuentra su razón en Cristo, que es el pleroma de todo el universo. Por tanto, el gran diseño no contiene solo el universo. Es la historia de la salvación de la sociedad humana y del cosmos.

\section{Bibliografía}

Aristóteles (1973) Obras completas, Madrid: Editorial Aguilar.

Astier, André (1967), "El científico frente a su propia fe" en Selecciones de teología, no. 24, Vol. 6.

Barth, Karl (200), Carta a los Romanos, Madrid: Biblioteca de Autores Cristianos.

Boff, Leonardo ( 2003), La voz del arco iris, Madrid: Editorial Trotta.

(2004), Ética y moral. La búsqueda de los fundamentos, Santander: Ed. Sal terrae.
Bunge, Mario (1970), La investigación cientifica, Madrid: Ed. Taurus.

Capra, Fritjof y Steindl-Rast (1993), Pertenecer al universo. La nueva ciencia al encuentro de la sabiduría, Buenos Aires: Ed. Planeta.

Camus, Albert (1960), Bodas, Buenos Aires: Ed. Sur.

Collins, Francis (2008), ¿Cómo habla Dios?, Bogotá: Editorial Planeta

Courault, Gustavo (2008), “Astrónomos descubren la galaxia más distante hasta ahora conocida", en Universia (Universidad Católica de Chile), 25,1, 2008.

Chardin de, Pierre T. (1965), El Fenómeno humano, Madrid: Ed. Taurus.

Folari, Roberto (2000), Epistemología y sociedad, Santa Fe: Ed. Lux.

Haught, John F. (2009), Cristianismo y ciencia: Hacia una teología de la naturale$z a$, Santander: Ed. Sal terrae.

Hawking, Stephen and Mlodinow, Leonard (2010), The grand design, New York: Bantan Books.

Hawking, Stephen (2005), Breve historia del tiempo, Barcelona, Ed. Crítica.

2008, Teoría del todo, el origen y el destino del Universo, México D.F.: Ed. Random Hause Mondadori.

(2003), El universo en una cáscara de nuez, Barcelona: Egedsa.

Hawking, S. y Penrose, R., (1996), La naturaleza del espacio y del tiempo, Madrid: Ed. Debate.

Heisenberg, Werner (1994), La imagen de la naturaleza en la física actual, Buenos Aires: Editorial Planeta. 
Juan Pablo II (1998), Fides et ratio, Ciudad del Vaticano: Librería editrice vaticana.

Kuhn, Tomas (1995), La estructura de las revoluciones científicas, México D.F.: Fondo de cultura económica.

(1994), La revolución copernicana, Madrid: Ed. Planeta.

Levinas, Marcelo Leonardo (2006), Las imágenes del universo: una historia de las ideas del cosmos, Buenos Aires: Ed. Siglo XXI.

Lightman, Allan P. (1995), Grandes ideas de la física, Madrid: Ed. McGraw-Hill

Morin, Edgar, (2006), El método. El conocimiento del conocimiento. Madrid: Ed. Cátedra.

Oerter, Robert (2008), La teoría de casi todo, México D.F.: Fondo de cultura económica.

Peacocke, Arthur, (1993), Teología para una era cientifica, Ed. Augsburg Fortress.

(2008), Los caminos de las ciencias hacia Dios, Santander: Ed. Sal Terrae.
Platón, (1979), Obras completas, Madrid: Editorial Aguilar.

Rahner, Karl (1967), Escritos de Teología, Tomos III y V, Madrid: Ed. Taurus.

Sagan, Carl, (1984), El cerebro de broca. Reflexiones sobre el apasionante mundo de la ciencia, México D.F.: Ed. Grijalbo.

(1998), Millones de millones, Barcelona: ed. Liberduplex.

(1997), Cosmos, Madrid: Talleres gráficos Soler.

Tomás de Aquino (1959), Suma Teológica, Ed, Biblioteca de Autores Cristianos.

Toro y Llaca, Carmen, (1999), Astronomía: Historia y calendario, Madrid: Ed. Instituto de Astronomía y Geodesia (UCM).

Von Weizsacker, Carl (1974), La imagen fisica del mundo, Madrid: Biblioteca de autores cristianos.

Von Rad, Gerhard, (1982), Teología del Antiguo Testamento, Salamanca: Ed. Sígueme.

Wicks, Jared, (2001), Introducción al método teológico, Navarra: Ed. Verbo Divino. 
